\title{
Urgences
}

\section{Un espace protocolaire}

\section{Le roi du monde de Régis Franc}

\section{Yves Lacroix}

Numéro 32, mai 1991

Lectures de bandes dessinées

URI : https://id.erudit.org/iderudit/025650ar

DOI : https://doi.org/10.7202/025650ar

Aller au sommaire du numéro

Éditeur(s)

Urgences

ISSN

0226-9554 (imprimé)

1927-3924 (numérique)

Découvrir la revue

Citer cet article

Lacroix, Y. (1991). Un espace protocolaire : Le roi du monde de Régis Franc.

Urgences, (32), 82-94. https://doi.org/10.7202/025650ar

Ce document est protégé par la loi sur le droit d'auteur. L'utilisation des services d'Érudit (y compris la reproduction) est assujettie à sa politique d'utilisation que vous pouvez consulter en ligne.

https://apropos.erudit.org/fr/usagers/politique-dutilisation/
Cet article est diffusé et préservé par Érudit.

Érudit est un consortium interuniversitaire sans but lucratif composé de l’Université de Montréal, l'Université Laval et l'Université du Québec à Montréal. Il a pour mission la promotion et la valorisation de la recherche. https://www.erudit.org/fr/ 


\section{Un espace protocolaire. Le roi du monde de Régis Franc}

\section{Yves Lacroix}

Des récits courts de Régis Franc, nous intéresse ici la recherche formelle de leur narration, non pas une recherche de singularité pour chacun des récits mais le choix préalable de quelques types de fragmentation de la planche, repris et modulés de façon plus ou moins régulière, comme si les formes cherchaient une adéquation parfaite au raconté.

Ainsi en est-il de l'album Le marchand d'opium ${ }^{1}$, composé de quatorze récits, tous de quatre planches, sauf un de trois planches intitulé comme par hasard Enjoy golden triangle. Sept de ces récits ont une structure commune ${ }^{2}$. L'organisation visuelle des autres est variable, mais la narration de chacun procède d'un principe formel qui trouve dans l'ordre du raconté une équivalence plus ou moins réussie. Nous avons déjà proposé une description de l'un d'eux ${ }^{3}$, nous nous occuperons cette fois du Roi du monde 4 (figure 1, p. 83, figure 2, p. 84 , et figure 3, p. 85) dont les diégèses foisonnent tout autant.

Le principe narratif de ce récit avait déjà caractérisé $H o n g$ Kong, terre de contrastes ${ }^{5}$ que Benoît Peeters a décrit ${ }^{6}$ en

1 Le marchand d'opium, coloriage de Sophie Charlotte Capdevielle, Paris, Dargaud, 1984.

2 "Marchand d'opium ", "La fuite en Chine ", "Rossignol et Bengali à Barcelone", " 11 heures du matin en plein été ", " Le point du jour ", "La soucoupe volante " et « Le sommeil ". A quelques dètails près, deux récits de Nuits de Chine (Dargaud, 1982) se conformaient déjà à cette matrice narrative: $\propto$ Trois couples en hiver au soleil " et "Le petit Futez".

3 Yves Lacroix, "Une écriture panoptique", dans Bande dessinée, récit et modernité, sous la direction de Thierry Groensteen, Paris, Futuropolis, 1988, p. 139-156.

4 «e roi du monde", daté de juillet 1983, paru dans Pilote $n^{\circ} 112$, septembre 1983, repris dans Le marchand d'opium, p. 26-29.

5 * Hong-Kong, terre de contrastes ", daté d'octobre 1976, paru dans Pilote, $n^{\circ} 33$, février 1977, puis dans Histoires immobiles et récits inachevés, Dargaud, 1977 , p. 39-43.

6 Benoit Peeters, "Une exploration interrompue", dans Les cahiers de la bande dessinée, $n^{\circ}$ 57, Grenoble, avril-mai 1984, p. 24-26. Un premier exposé schématique avait permis d'illustrer la catégorie de "mise en page" que Peeters a qualifiée de "productrice" (* Les aventures de la page", dans 


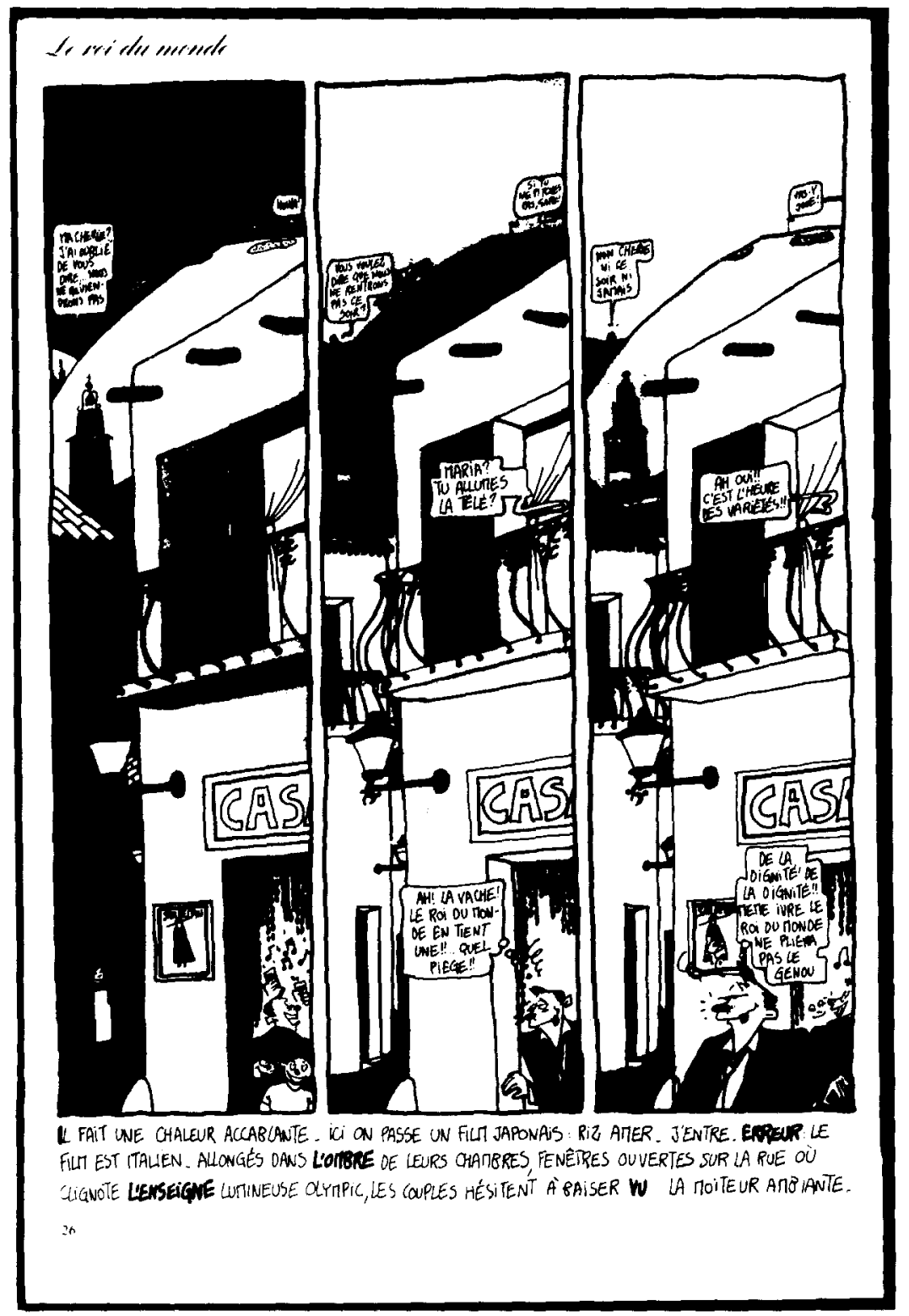

Figure 1 


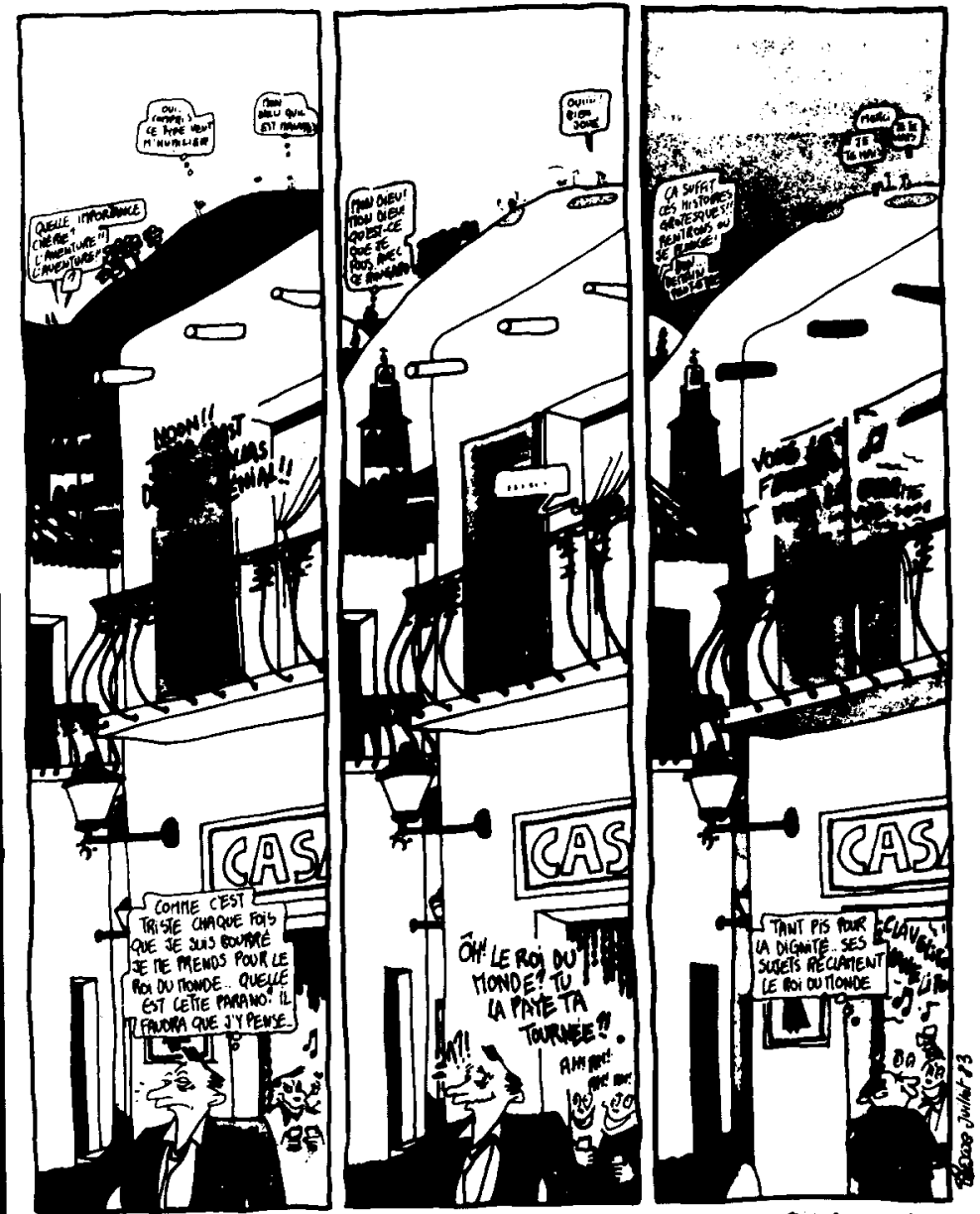

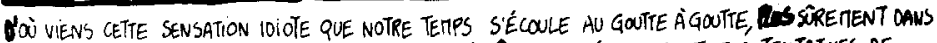
ChAQUE FAUTE COTTISE? LA CHALEUR, QUI ENGOUROT 4 TEE, RENO EPUISANTE TOUTES TENTATIVES DE

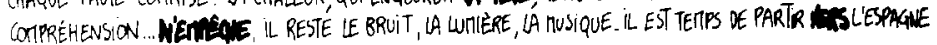



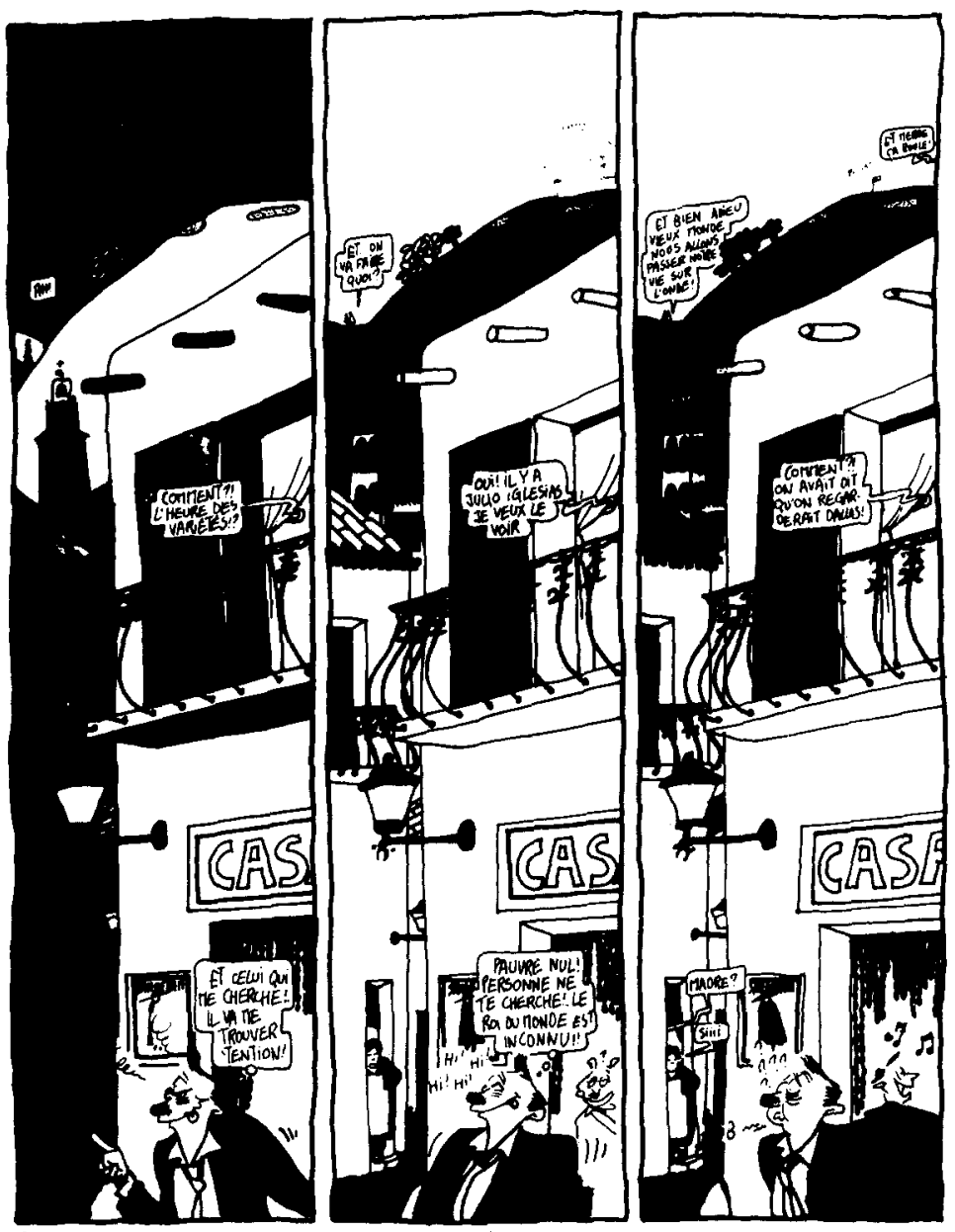

ET PUIS, QUELQU'UN BRT OES TAINS. ASSSS SUR UNE VOITURE EN DÉCOTPOSITION, UN GWUEE OE NEGRES RYTHTE

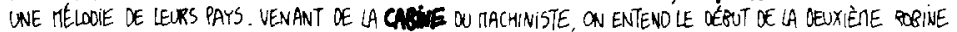

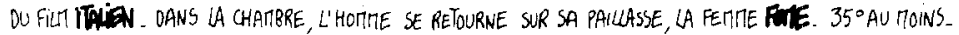




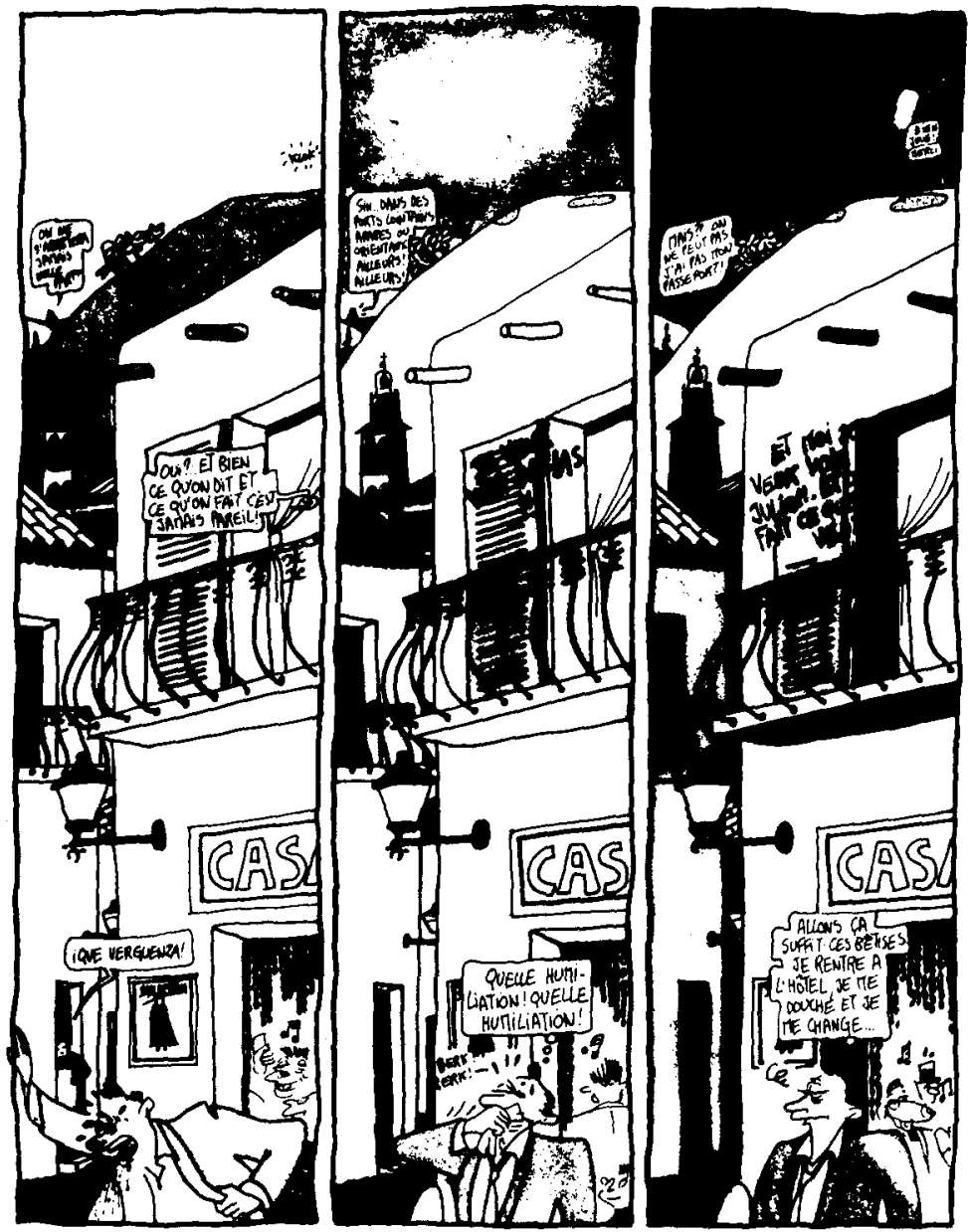

NORS, LE MACHINISTE SORT DE SA CABINE ET CONTETPLE IA RUE and IL ALUITE UNE ClGARETEE ET TIRE DESSUS. LA FUMEE, QUI SECOMIE OE SA BOUCHE, SENFONCE DANS LA NUTT. PAS UN BRIN DOXYGENE,

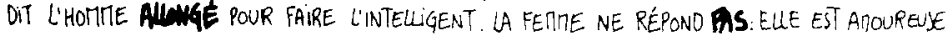
ix 
1984 avec une pertinence dont nous avons fait notre profit: douze vignettes verticales semblables, distribuées régulièrement sur quatre planches, racontent parallèlement un certain nombre d'histoires autonomes ${ }^{7}$. Elles étaient trois dans le premier récit, elles sont cinq dans Le roi du monde, si on tient compte du récitatif qui n'existait pas dans le premier cas ${ }^{8}$.

Quel est l'enjeu de cette simplicité visuelle dans une narration aussi manifestement parataxique?

Sa fragmentation apparaît d'emblée comme une subversion des canons de la bande dessinée ${ }^{9}$. La planche est divisée en trois bandes verticales, rompant avec la lecture horizontale coutumière. Même si cette verticalité est offerte d'un seul tenant, on pourrait déclarer que chaque unité narrative globale est fragmentée en trois vignettes non cadrées: celle de la rue au bas de l'image, celle de la fenêtre en son centre et celle de l'horizon tout en haut. Mais la subversion véritable de cette composition réside dans le fait qu'à la lecture verticale, tout de même canonique, ne correspond aucune continuité événementielle. En effet, aux trois espaces superposés correspondent trois diégèses hétéroclites. C'est la lecture qui révèle, dans la répétition du même, des développements syntagmatiques horizontaux dont l'axe est contredit par le montage d'une planche qui devrait en diriger l'ordonnance.

Transgressive autrement, et depuis les commencements de son exercice de la bande dessinée, apparaît chez Régis Franc la juxtaposition de scènes brèves dont le foisonnement n'a jamais été aussi important.

Conséquences, $n^{\circ} 1$, Paris, automne 1983, p. 38-43). Légèrement modifié, ce dernier texte a fait l'objet d'une communication à Montréal en 1985 ("Comment lire une BD?", dans Actes, premier colloque de bande dessinée de Montréal, sous la direction de Jacques Samson et André Carpentier, Montréal, Analogon, 1986, p. 209-212).

7 De 1977 à 1984, trois autres récits ont constitué des variables de cette narration: « Un dimanche d'été " (Nouvelles histoires, Dargaud, 1978), "Le bordel de Madame Antoine" et * Le roi de la jungle" (Souvenirs d'un menteur, Dargaud, 1979).

8 Nous pourrions parler de six anecdotes si nous prenions en compte les épiphanies de la dame en noir dans le cadre de sa porte, dans le coin gauche inférieur des vignettes. Nous pourrions même prétendre qu'il y en a plus, à détailler l'ubiquité du récitant.

9 II en est de mème de « L'ananas des iles " Le marchand d'opium, p. 1720). Voir Yves Lacroix, loc. cit., p. 150-153. 


\section{Observation I: la valeur locutoire de la narration}

Nous pensons d'abord à la valeur locutoire 10 d'un récitatif posé directement sur le blanc de la page, instance d'un narrateur qui commenterait le texte iconique.

Il s'agit proprement d'un métarécit, moins caractérisé par un changement de niveau par rapport à un récit tuteur, que par une référence à un récit premier qui serait désigné par le titre. On pourrait le dire également des histoires «superposées " de ce texte: elles commentent toutes un CAS ${ }^{11}$, celui d'un pauvre type qui se prend pour le roi du monde.

Avec la légende qui supporte et lie la triple image sur toute la largeur de la planche, nous sommes dans le lieu de la production du récit, le lieu de sa récitation graphique. L'inscription de cet énoncé sur la page fait ressortir le caractère calligraphique de toutes les énonciations linguistiques, de même que le font les cadres des vignettes anormalement tirés à main levée, et désigne les énoncés comme autant de traces du geste scripteur.

À la brièveté de l'esquisse ne manquent même pas la négligence du trait, et les distractions d'une transcription linguistique trop rapide ${ }^{12}$.

10 Un acte de parole est dit locutoire dans sa fonction d'articuler et de combiner des sons, «dans la mesure aussi où [il] évoque et relie syntaxiquement les notions représentées par les mots " (Oswald Ducrot et Tzvetan Todorov, Dictionnaire encyclopédique des sciences du langage, coll. Points, $n^{\circ} 110$, Paris, Seuil, 1979, p. 428).

11 Bien que ce lexème soit dans toutes les vignettes au-dessus de la tête du personnage, aussi évident que le nez au milieu de son visage, je dois à André Carpentier de me l'avoir désigné. Quoi que nous en écrivions, l'ordre des récits n'est pas assuré. Si le récitatif (dont la lettre est plus haute que celle des autres énoncés linguistiques) et la séquence qui apparaît à l'avant-plan de l'image (privilégiée par la déixis du titre) devraient correspondre aux récits premiers, il faut bien noter cependant que c'est en haut de la première image qu'il se passe d'abord quelque chose (bien que ces événements soient voilés par l'éloignement et leur mise hors champ) alors que le protagoniste de l'avant-plan n'est pas encore désigné dans le champ inférieur de l'image. On pourrait affirmer que chacune des histoires parallèles " interprète " toutes les autres confondues.

12 Dans la troisième vignette de la deuxième planche (séquence du voilier), il est écrit «ET BIEN" pour «EH BIEN"; dans la deuxième vignette de la quatrième planche (séquence du roi du monde), il est écrit * TU LA PAYE [Sic] TA TOURNEE ? " ; également dans le récitatif de la dernière planche, il est écrit * D'OÜ VIENS [sic] 
Nous inscrivons dans le prolongement de cette observation l'application arbitraire des couleurs, immotivée bien sûr par rapport au référent réel, mais tout de même structurée dans son organisation graphique. D'une part on peut noter la permanence de certaines couleurs qui assurent une stabilité à l'ensemble ${ }^{13}$. D'autre part, trois teintes d'une même couleur marquent dans chaque vignette la distance des trois maisons superposées, du plus pâle au plus foncé; et les ombres sur les murs ont toujours la teinte plus sombre du motif architectural le plus éloigné. Finalement, le ciel reprend toujours la teinte de ce dernier motif.

Bien que l'immuabilité de la perspective impose dans toutes les vignettes la présence d'un point immobile d'ocularisation externe, quelque part en avant de la porte de la CASA, cette visée est incompatible avec la composition de l'ensemble. En effet, il est invraisemblable que la ligne des eaux apparaisse alors par-dessus les toits.

Ce que nous tentons de faire valoir ici, c'est le travail de l'énonciation: la continuité de «la monstration" - pour éviter de dire "la représentation " - dévoile deux champs visuellement incompatibles. Leur inscription dans un même espace graphique ne peut signifier que leur simultanéité. C'est l'énonciation ici qui impose la congruence de l'espace, non la consécution événementielle, non plus la cohérence d'un éventuel référent réel.

\section{Observation II: le caractère sériel de la narration}

Quels champs sémantiques justifient la juxtaposition verticale de ces esquisses? Quels sont leurs équivalents dans la sériation horizontale des vignettes?

Nous trouvons dans le récitatif un programme métatextuel:

CETTE SENSATION..." " puis \& REND EPUISANTE TOUTE TENTATIVES [sic] DE COMPREHENSION". On pourrait ajouter à ces distractions l'absence d'arbres sur la colline de la troisième vignette de la première planche.

13 Soit la couleur invariable du pavé; celles du balcon, des volets et du rideau de la chambre; celles des intérieurs; celles du personnage qui se prend pour le roi du monde; celles de la femme qu'un enfant appelle "MADRE"; celles des affiches posées sur le mur de l'avant-plan; celle de la mer triangulaire à la pointe du clocher. 
IL FAIT UNE CHALEUR ACCABLANTE. ICI ON PASSE UN FILM JAPONAIS: RIZ AMER. J'ENTRE. ERREUR: LE FILM EST ITALIEN. ALLONGES DANS L'OMBRE DE LEURS CHAMBRES, FENETRES OUVERTES SUR LA RUE OU CLIGNOTE L'ENSEIGNE LUMINEUSE OLYMPIC, LES COUPLES HESITENT A BAISER VU LA MOITEUR AMBIANTE (planche 1). ${ }^{14}$

Grâce à une confusion momentanée sur les lieux décrits de la dernière phrase, la focalisation interne initiale éclate en une focalisation zéro équivalente à la narration panoptique des images. Il n'existe aucune consécution diégétique, depuis la salle de cinéma où entre la première personne, par le film italien dont cette personne est curieuse, par les couples qui baisent dans les chambres (celle singulière de Riz amer et celles, multiples, du quartier), parmi lesquels une femme fume auprès d'un amant indifférent, puis par les nègres sur la place qui rythment "UNE MÉLODIE DU PAYS», jusqu'au projectionniste qui sort de sa cabine pour fumer.

Cette absence de congruence dans le syntagme narratif laisse place à une collocation paradigmatique dont les réseaux principaux seraient une immobilité torpide et une culpabilité confuse, ainsi que le propose le quatrième paragraphe du récitatif, dans un énoncé juste assez grandiloquent pour qu'on soupçonne le pastiche:

D'OUं VIENS [sic] CETTE SENSATION IDIOTE QUE NOTRE TEMPS S'ÉCOULE AU GOUTTE A GOUTTE, PLUS SÛREMENT DANS CHAQUE FAUTE COMMISE? LA CHALEUR ENGOURDIT LA TÊTE, REND ÉPUISANTE TOUTES TENTATIVES [sic] DE COMPRÉHENSION...

Ces isotopies sont déployées dans les esquisses dessinées et les motifs principaux y sont ceux

- de l'évasion (qui caractérise les mâles)

vers DES PORTS LOINTAINS (voilier, vignette 8),

vers le Texas de la télévision américaine,

14 On ne manquera pas d'observer que certains mots, parfois une lettre isolée, sont en caractères gras. Franc s'en est expliqué à Thiemy Groensteen: "Les mots écrits en gras rythment le texte. Généralement j'en mets deux sur la première ligne, un sur la deuxième, deux sur la troisième, etc. Parfois je choisis certains mots plutōt que d'autres mais quand je n'ai pas le temps, je prends n'importe lesquels." (Les cahiers de la bande dessinée, $n^{\circ} 57$, p. 9.) 
vers l'Espagne, celle de Julio Iglesias, celle d'une CASA fréquentée par un roi et qui pourrait bien être de ce fait un "château en Espagne", pays vers lequel aspire le récitant: IL EST TEMPS DE PARTIR VERS LESPAGNE (vig. 12);

— de la velléité (qui caractérise les mâles encore une fois);

- de l'immobilité, celle du voilier sur la ligne de l'horizon et celle de la balle de golf qu'il faut "PITCHER ", c'est-à-dire frapper de telle sorte qu'elle ne roule pas sur le gazon et ne risque pas de s'ensabler;

- de l'humiliation, surtout dans la séquence du roi du monde,

depuis la DIGNITÉ réclamée pour le héros (vig. 3),

par le QUE VERGUENZA! infamant de la mère (vig. 7),

par l'HUMILIATION constatée du roi (vig. 8),

jusqu'à sa défaite définitive: TANT PIS POUR LA DIGNITÉ (vig. 12);

mais aussi l'humiliation du golfeur maladroit: CE TYPE VEUT M'HUMILIER (vig. 10);

avec la paranoīa comme motif complémentaire, celle du golfeur peut-être, mais surtout celle explicitée du roi du monde: QUELLE EST CETTE PARANO? (vig. 10).

Il est aussi d'autres récurrences plus formelles qui agissent comme des marques de la sérialité:

- le SIIII condescendant de la mère (vig. 6) et celui du marin "ringard" (vig. 8), comme le OUIIII! du golfeur paternaliste (vig. 7);

- la chaîne phonique de PORT (voilier, vig. 8) et de PASSEPORT (voilier, vig. 9), et pourquoi pas le PORTO de Sandeman!

Et pourquoi ne pas prendre en compte un jeu de golf à proximité d'un golfe qui est une «partie de la mer qui avance dans les terres (Larousse)"?

Et pourquoi ne pas rapprocher l'existence d'une trappe de sable où s'enlisent les balles de golf, et la représentation de 
92

l'«homme de sable" sur le mur de la CASA? Et pourquoi ne pas rapprocher cette trappe du PIEGE dans lequel s'enivre le roi du monde (vig. 2)?

Et, puisque dans le récitatif le lexème RIZ du titre $R I Z$ $A M E R$ est désigné pour une fausse destination (UN FILM JAPONAIS) qui ne manque pas pour autant d'évoquer un ailleurs, pourquoi ne pas y lire le mot RIS qui signifie «chacune des bandes horizontales des voiles, qu'on replie [...] pour diminuer la surface de voilure présentée au vent (Robert)"? Une fois partis, pourquoi ne pas en discriminer le phonème MER?

Et pourquoi ne pas associer la mère épisodique dans le cadre de sa porte (MADRE, vig. 6) à la MER océane dans son triangle supérieur?

Une fois livrés à la frénésie du paradigme, pourquoi nous arrêter?

Notons que Georges Sadoul écrit que Riz amer raconte l'impatience d' '...une mondaine à la tête tournée par la presse du cœur". L'historien précise que cette littérature est celle des "fumetti ", qui est le terme italien pour dire «bande dessinée"; il écrit que l'héroïne vit "dans la boue jusqu'aux cuisses, mais la tête perdue dans une usine à rêves " 15 , encore qu'il n'y ait pas l'ombre d'une bande dessinée dans le film de De Santis.

Et puisqu'il a été question de rêves, notons que «sand man" signifie "the man who, in folklore, puts sand in the eyes of children to make them sleepy (Random)", et que, si nous en croyons le Robert, « le marchand de sable " en langage enfantin serait «quelqu'un qui a sommeil»: le marchand de sable est passé, c'est-à-dire qu'on a déposé sur les paupières du sable qui les appesantit.

Mais à cause de l'enseigne lumineuse du cinéma OLYMPIC qu'on aperçoit par la fenêtre d'une chambre, dans le récitatif de la première planche, rappelons aussi que, dans le conte de Hoffmann Der Sandmann (L'homme au sable), c'est par une fenêtre que l'étudiant Nathanaël découvre une automate nommée "Olympia", dont il tombe amoureux. Et souvenons-nous

15 Georges Sadoul, Dictionnaire des films, Paris, Seuil, 1981, art. " Riz amer ". 
que ce conte servit d'exemple à l'exposé de Freud sur l'inquiétante étrangeté et qu'il voyait dans le personnage de l'homme au sable «le père redouté, de la part de qui on craint la castration " ${ }^{16}$. Notons que l'image de l'homme mythique est accolée à la maison de débauche, comme pour affirmer sur elle son autorité: celui qui voudrait bien être le roi du monde et qui se trouve là comme dans un château en Espagne, s'y trouve dans sa dérive comme dans la maison de l'homme au sable.

\section{Observation III: le protocole de la première vignette par laquelle nous aurions pu commencer}

Dans la première vignette, les champs des trois diégèses de droite sont décadrés, celui de la casa, celui de la fenêtre et celui du golf, alors que dans le coin supérieur gauche la "scène" du voilier est effacée par son éloignement. Les golfeurs seront à peine distincts quand ils entreront dans le champ de la cinquième vignette. Ne sont vraiment visibles que la mère périodique dans le coin gauche inférieur, et, dès la deuxième vignette, le personnage qui se prend pour le roi du monde. Ainsi que l'écrirait Michel Chion ${ }^{17}$, trois des quatre dialogues de ce récit sont acousmatiques, c'est-à-dire que la source des voix est dissimulée au regard.

Comme pour la narration verbale du récitatif, la focalisation de la narration iconique est au point zéro, c'est-à-dire qu'elle n'existe pas, résolument à l'extérieur des lieux mais capable d'entendre ce qui se dit dans la tête d'un personnage, à l'intérieur des chambres et sur la ligne d'horizon.

Cependant, la lecture de cette vignette prototype est structurée par trois regards surcadrés de la diégèse inférieure, d'abord celui de la mère dans sa porte et celui de l'enfant dans la porte de la casa, qui portent approximativement hors cadre un peu en avant du premier plan. À moins que le regard de la mère ne porte sur l'enfant! Les positions de ce paradigme familial ne sont pas incompatibles.

16 Sigmund Freud, "L'inquiétante étrangeté ", traduit par Marie Bonaparte et Mme E. Marty, dans Essais de psychanalyse appliquée, coll. "Idées ", Paris, NRF/Gallimand, 1978, p. 182.

17 Michel Chion, La voix au cinéma, Paris, Cahiers du cinéma/Éditions de l'Étoile, 1982, particulièrement le chapitre intitulé * L'acousmètre", p. 25-33. 
94

Le troisième regard est induit par analogie, c'est celui de Sandeman dans le cadre de son affiche, dans l'axe vertical de l'image. À moins que la mère ne regarde vers Sandeman qui regarde vers l'enfant ou vers un ocularisateur externe à partir duquel la scène est structurée! C'est dans l'ambivalence de cet avant-plan que vient s'installer le soûlard dans la deuxième vignette à la place de l'enfant, alors que sont dissimulés les regards des figures autoritaires. Ces derniers retrouvent leurs droits dans la vignette 7 , alors que la mère stigmatise le comportement du jeune homme (QUE VERGUENZA!), lequel s'approprie l'infamie dans la vignette suivante: QUELLE HUMILIATION!

Mais revenons à notre point de visée. C'est à partir de celui-là, d'abord en creux, qu'est structurée la double monstration d'une verticale "acousmatique" à droite (les couples de la chambre et du terrain de golf) et d'une verticale "familiale" à gauche, depuis la mere dans le premier champ, entre les maisons du village, par le beffroi dressé, jusqu'à la mer supérieure.

On pourrait induire de cette observation l'hypothèse que l'organisation de l'espace est ici inspirée par le fantasme de la scène primitive, quelque scène familiale. Mais là n'est pas notre propos.

Il nous intéresse de conclure ici que n'est montré dans ce récit que l'espace entre les diégèses, à la fois une continuité et une distance qui fondent leur disparité, dans un énoncé panoptique répétitif, qui est le lieu graphique de leur collection. Là où se trouve ordinairement représenté ce qui semble être le signifié obligé de la bande dessinée, c'est-à-dire l'histoire, on ne trouve ici qu'un programme de lecture, une mise en place de la structuration du sens, un protocole de lecture pour un carnet d'esquisses. 